\title{
PRESENTACIÓN
}

La Revista InterSedes se complace en presentar un nuevo número de manuscritos, todos ofreciendo una muestra del trabajo investigativo y docente que en nuestra alma mater se lleva a cabo.

En esta ocasión, las contribuciones provienen de Guanacaste (Olivares, Bermúdez), Turrialba (Castillo), Heredia (Elizondo y Monge), San José (Díaz, Solano y Amador, Chang, Aragón) y Argentina (Sandí y Cruz). La mayoría de las contribuciones provienen de estudios de posgrado, proyectos de investigaciones y estudios interinstitucionales. Otros provienen de un análisis crítico del trabajo docente.

La temática representada en el presente número amplía los horizontes a la relación entre la fe religiosa y la ciencia (Aragón) un tema muy presente en nuestro campus universitario, al patrimonio cultural intangible costarricense (Chang). Los resultados de investigaciones en materia de educación (Olivares, Sandí y Cruz) no faltan con sus aportes para el mejoramiento de los procesos de aprendizaje y enseñanza. El estudio del clima del Caribe y su registro histórico (Díaz, Solano y Amador), una reflexión historiográfica de la composición musical (Castillo). Finalmente, la producción agrícola (Elizondo y Monge), así como los procesos para la toma de decisiones propias de las Pymes (Bermúdez).

Como es de todos y todos conocidos, una publicación periódica requiere de aportes colectivos como los pares evaluadores y los Consejos Editoriales y Científicos, pero también de los criterios e indicadores de evaluación. En nuestro caso, UCRIndex recientemente ha planteado nuevos retos con otros indicadores que garanticen la calidad científica de las publicaciones de la Universidad de Costa Rica. En consecuencia, el trabajo editorial de este año se intensifica, y se espera que los resultados finales sean los esperados por la comunidad universitaria.

Sin más que anotar, el aporte de la Revista InterSedes a la producción científica está en sus manos.

M. Ph. Jimmy Washburn

Director, Revista InterSedes 


\section{EFECTO de LOS SERVICIOS CONTABLES EN LA TOMA DE DECISIONES DE LAS PYMES}

The Financial Services’ EfFects on Decision-MAKing Process of SMeS

LUIS ALBERTO BERMÚDEZ CARRILLO ${ }^{1}$

\begin{tabular}{|l|l|}
\hline Recibido: 7 de abril del 2015 & Aprobado: 7 de abril del 2017 \\
\hline
\end{tabular}

DOI: http://dx.doi.org/10.15517/isucr.v18i37.28653

\section{Resumen}

El propósito del presente artículo es analizar el efecto que tiene para la toma de decisiones de las pymes contratar servicios contables. Para el desarrollo de la investigación se planteó la pregunta en relación a, qué tanto ayuda a la toma de decisiones de las pymes el contratar los servicios contables en lugar de desarrollarlos dentro de la empresa. Para el análisis se consideraron las variables: tipo de actividad comercial y económica que desarrollan, el tipo de servicio e información contable que demandan, las capacidades técnicas que tienen para entender y extraer de la información contable datos relevantes que se apliquen a la toma de decisiones y los criterios por los cuales contratan servicios contables. Como muestra para la realización del estudio, se trabajó con 61 pymes, todas ellas contratan servicios contables; la muestra fue seleccionada intencionalmente por la naturaleza de la pyme y por su ubicación. Los resultados obtenidos reflejan que las pymes contratan servicios principalmente para la presentación de los reportes e informes tributarios, carecen de formación académica y técnica para poder hacerles frente a los procesos contables, conocen los estados financieros, pero no saben interpretarlos, contratan servicios contables por precio, confianza y puntualidad. Algunos motivos por los cuales no realizan su propia contabilidad son la falta de capacitación y la falta de recursos económicos.

Palabras claves: Servicios contables, toma de decisiones, control, actividad comercial, capacitación, pymes.

${ }^{1}$ Profesor de Dirección de Empresas. Principios de Administración, Contabilidad y Finanzas, Sede de Guanacaste, Universidad de Costa Rica, luis.bermudez@ucr.ac.cr 


\begin{abstract}
The purpose of this paper is to analyze the effect of making decisions for SMEs to hire accounting services. For the development of research question was raised regarding what helps both decision making SMEs hiring accounting services rather than develop them within the company. type of trade and economic activities performed, the type of service and accounting information demand, technical capabilities they have to understand and extract accounting information relevant data to be applied to decision-making: For analysis variables were considered and the criteria by which hire accounting services. As a sample for the study, we worked with 61 SMEs, all hire accounting services; the sample was selected intentionally by the nature of the SME and its location. The results show that SMEs hire services primarily for filing reports and tax reports, lack of academic and technical training to cope with the accounting processes, know the financial statements, but do not know interpret, hire accounting services by price, reliability and punctuality. Some reasons for not doing their own accounting are lack of training and lack of economic resources.
\end{abstract}

Key words: accounting, decision making, control, business, training, SMEs.

\section{INTRODUCCIÓN}

En los últimos años las pymes han contribuido de manera importante en el desarrollo productivo de Costa Rica. Con ellas se ha generado una nueva forma de visualizar y de hacer negocios, y esto ha tenido un efecto importante en el desarrollo local y nacional del país. Por ende, el Gobierno ha propuesto iniciativas de fortalecimiento y apoyo reflejados en programas como: Banca de Desarrollo, proyectos del Ministerio de Economía, Industria y Comercio; capacitaciones por medio de universidades públicas, capacitaciones que imparte el Instituto Nacional de Aprendizaje, entre otros. Todas estas iniciativas motivan a las pymes a fortalecer sus proyectos en materia comercial y a buscar nuevas formas de ampliar su conocimiento. Sin embargo, la preocupación número uno en las pymes sigue siendo la de sus recursos limitados, además de muchos otros problemas, como la falta de instalaciones, sistemas de administración inapropiados para su operación, falta de capacitación en diversos campos de la administración, y la limitación en su desarrollo en aspectos técnico-profesionales; todo lo cual se origina en la falta de recursos, porque para las pymes es muy difícil asumir costos de capacitación y adiestramiento. A pesar de que ellas gozan de algunos beneficios en materia tributaria están obligadas al pago de impuestos. Esta contribución se calcula y presenta, en la mayoría de los casos, de manera independiente, por medio de un contador o de un despacho contable y, por lo general, quienes hacen este tipo de trabajo son los contadores independientes, externos a la empresa. 
Según ese planteamiento, lo que interesa en este artículo es analizar qué tan importante es la contabilidad para la toma de las decisiones empresariales y cuánto afecta el hecho de que la contabilidad no se realice en lo interno de la empresa. Aunque financieramente el pagar por los servicios contables significa un beneficio por ser estos de costos menores, en el entorno empresarial se pueden presentar también muchas desventajas.

Sobre el aspecto que se analiza, específicamente en el ámbito costarricense no se encontraron estudios; sin embargo, el análisis se centra en el esfuerzo de entender cuál es la relación que tiene la contabilidad contratada a terceros con la toma de las decisiones empresariales.

Planteado así el fin de la presente investigación, se proponen ahora los siguientes objetivos:

- Determinar el tipo de actividad comercial y económica que desarrollan las pymes que recurren a los servicios contables independientes;

- Reconocer el tipo de servicio e información contable que demandan las pymes de las oficinas contables;

- Identificar las capacidades técnicas contables que poseen los colaboradores y propietarios de las pymes que utilizan servicios contables independientes;
- Identificar cuáles son los criterios que privan en las pymes para contratar un servicio contable.

Lo que tiene el mayor interés en esta investigación es conocer cuáles son los criterios y razones que privan en las pymes, en las que lo hacen, al contratar servicios contables en lugar de generarlos en la propia empresa.

\section{MARCO TEÓRICO}

La actividad económica puede denotarse como aquella acción empresarial en la que se intercambian o comercializan bienes y servicios para satisfacer las necesidades de las empresas y del mercado. La actividad comercial permite la generación de riqueza mediante la transformación de los recursos naturales.

Internacionalmente existe una clasificación de las actividades económicas basada en diferentes estadísticas económicas, tales como población, producción, empleo, ingreso nacional, y otras estadísticas de mercado. Dicha clasificación se denomina "Clasificación Industrial Internacional Uniforme, la cual divide la economía en actividades principales, secundarias y auxiliares. (Naciones Unidas, 2002, pp. 5-7).

En dicha clasificación lo que se busca es agrupar diferentes actividades económicas específicas y valorarlas por su relación con la actividad operativa de las empresas que las realizan en un país específico. 
Para tener una referencia más clara de la clasificación de las actividades económicas, Ayllón Torres (2004) hace la siguiente explicación:

- Actividades primarias, que estén estrechamente relacionadas con las condiciones geográficas. Estas proporcionan las materias primas, que son: ganadería, minería, pesca, caza y silvicultura. Son las predominantes en países de escaso desarrollo.

- Actividades secundarias que pertenecen a la industria, o sea, a la transformación de materias primas, y que dan lugar a diferentes tipos de industria. Predominan en países desarrollados.

- Actividades terciarias, que son todas las ocupaciones en las que se prestan servicios como transporte, comunicaciones, comercio y turismo. (p. 17).

Muchas de las actividades predominantes en las pymes en Costa Rica se agrupan como primarias y terciarias. Sus actividades secundarias corresponden más a países desarrollados en los que la industrialización y la transformación de materia prima es la base de sus actividades comerciales. De igual manera, la complejidad que caracteriza a las empresas industriales en sus sistemas de producción y en la cantidad de colaboradores con los que cuentan no permitiría clasificarlas como pymes.

No obstante, la complejidad de la actividad económica de las pymes pareciera tener alguna relación con la centralización o desconcentración de los servicios contables. Independientemente del tipo de actividades que desarrollen $y$, en cumplimiento de la normativa costarricense relacionada con el rendimiento de cuentas y la presentación de informes a la Administración Tributaria, todas deben llevar contabilidades, ya sea que se produzcan propiamente en la empresa, o como servicios contratados a contadores $u$ oficinas contables independientes.

El término contabilidad se refiere a la actividad de llevar de forma clara y ordenada las cuentas de la empresa. En el aspecto empresarial, la contabilidad, además de permitir llevar un orden interno, debería ser una herramienta que permita controlar y tomar decisiones.

Según Horngren (2003), la contabilidad es:

El sistema de información que mide las actividades de las empresas, que procesa esa información en estados (informes) y comunica los resultados a los tomadores de decisiones. La contabilidad no equivale a la teneduría de libros, que es un procedimiento contable, de igual modo que la aritmética es un procedimiento matemático. Es frecuente que se llame "lenguaje de los negocios" a la contabilidad. Cuanto mejor se entienda ese 
lenguaje, tanto mejores serán sus decisiones de negocios y la administración de los aspectos financieros de su vida personal. (pág.

5).

Dada la definición anterior, se puede entender la contabilidad como un sistema para comunicar resultados de la empresa. Esos resultados deben ser comunicados a los tomadores de decisiones y deben estar estructurados en forma de estados e informes para que permitan la administración de los aspectos financieros de la compañía.

Todas las instituciones, empresas u organizaciones deben, por orden, control y mejores decisiones, tener sistemas contables y llevar sus contabilidades al día. La contabilidad se ha convertido en un idioma universal de los negocios y ello implica que las empresas tengan que capacitarse para producir información contable, o simplemente pagar para que sean otros los que la produzcan.

Al respecto Martínez (2009) refiere que "la contabilidad identifica, evalúa, registra y produce cuadros-síntesis de información. La contabilidad tiene como principal misión el proporcionar una información adecuada y sistemática del acontecer económico y financiero de la empresa". (p. 9).

Con base en el concepto mencionado, ese autor hace alusión a la contabilidad como un sistema de información que sirve como herramienta para la toma de decisiones; por tanto, y como plataforma de esos preceptos, se debe concebir la contabilidad como un proceso de ayuda y apoyo en la toma de decisiones. Sin embargo, pareciera ser que por lo menos en el ámbito de las pymes, para que se vea el efecto real de la información contable, es necesario que esta sea preparada en lo interno.

Bajo esa premisa es necesario revisar los tipos de servicio e informes contables que demandan las pymes de las oficinas contables. En general, los servicios contables están muy relacionados con los tipos de contabilidad requeridos por ellas. Por ende, en el presente estudio se va a hacer una distinción de los diferentes tipos de contabilidad que se generan en las empresas, entre ellos las contabilidades administrativa, financiera y fiscal.

La contabilidad administrativa se especializa en los informes internos y "mide, analiza y presenta información financiera y no financiera que ayuda a los gerentes a tomar decisiones para alcanzar las metas de una organización". (Horngren, Datar, \& Foster, 2007, p. 2).

Una de las características que se pueden identificar en la contabilidad administrativa es que los informes elaborados sirven para planear eventos futuros. Además, la contabilidad administrativa no está regulada por las normas contables y los informes son elaborados específicamente para circunstancias o necesidades concretas de la administración. También, por lo general la contabilidad administrativa tiene que ayudarse con algunas otras disciplinas de las ciencias sociales, como la estadística y la economía, entre otras. 
Por otra parte, la contabilidad financiera tiene como objetivo presentar información tanto en lo interno como en lo externo, pero los informes elaborados los preparan para personas externas a la empresa que de una u otra manera mantienen una relación de interés con ella, llámense esos terceros inversionistas entidades gubernamentales, bancos, proveedores u otros. (Horngren, Sundem, \& Elliot, 2000, p. 7).

Este tipo de contabilidad, a diferencia de la contabilidad administrativa, basa sus informes en normas contables que son dadas para analizar y evaluar eventos que ya han pasado. Tales informes están vinculados con las actividades de la empresa. Por ello es necesario saber qué tan rentable está siendo la empresa. La contabilidad administrativa es opcional y permite adaptarse a la naturaleza y necesidad de cada empresa.

El tercer tipo de contabilidad pareciera estar muy arraigado en la naturaleza de las pymes, pues esta tiene que ver con la presentación de los informes que solicita el Ministerio de Hacienda. Robles, Valdés \& Alcérreca, Joaquín (2000) reseñan que "la contabilidad fiscal es el área de la contabilidad que se encarga de dar cumplimiento a las obligaciones tributarias de la empresa. (p. 178).

Como las pymes son empresas de naturaleza pequeña por lo general no tienen dentro de su estructura organizacional un departamento $\mathrm{u}$ oficina propiamente de contabilidad; por tanto, deben recurrir a terceros para que las apoyen en la elaboración de sus informes financieros.

Respecto a los informes contables, estos se detallan en un idioma universal y actualmente están avalados y estructurados bajo las Normas Financieras Internacionales. Dichos informes deben reunir información básica y estándar, de tal manera que cualquiera pueda leerlos y entender su composición y su mensaje. Los informes contables persiguen dos objetivos, que la información sea fiable y que esté basada en principios y criterios generalmente aceptados. La información debe ser comparable para poder definir metas y controles. (Alcarria Jaime, 2009, p. 16).

No obstante, en referencia a la información contable que se elabora internamente, esta se trata a conveniencia y según la necesidad de cada empresa. No hay disposiciones legales ni de forma que regulen el tipo de informe que debe utilizar cada una. Sin embargo, cuando se trata de informes que van a ser utilizados externamente estos necesitan tener formatos obligatorios. Jaime Alcarria (2009) afirma que "los informes que elabora la contabilidad financiera están formados por cinco documentos, balance, cuentas de pérdidas y ganancias, estados de cambios en el patrimonio neto, estado de flujos de efectivo y memoria.” (p. 21). 
El balance es el informe que expone la situación de la empresa a una fecha determinada. Está compuesto por los recursos económicos propiedad de la empresa (activos), todas las deudas $\mathrm{u}$ obligaciones que tiene la empresa (pasivos) y su valor neto (activos menos todos los pasivos). Las cuentas de pérdidas y ganancias exponen y detallan la relación de ingresos y gastos que tuvo la empresa en un periodo determinado, y también visualiza las pérdidas o ganancias que ha tenido.

En el estado de cambio en el patrimonio necesariamente se necesita comparar dos periodos contables para lograr visualizar cambios importantes en las cuentas de los activos de la empresa. Para Eslava \& Marín (2009), el Estado de cambio en el patrimonio neto tiene como objetivo "informar de los cambios habidos en el patrimonio neto entre dos ejercicios mostrando los incrementos y decrementos en los activos netos (patrimonio neto) durante el ejercicio". (p. 66)

Respecto al estado de flujo de efectivo, este informa sobre la forma en que se utilizaron los activos monetarios, representados en forma de efectivo o equivalentes.

En referencia al estado de flujo de efectivo, Eslava \& Marín (2009) exponen:

Dentro de los objetivos del flujo de efectivo este pretende contribuir con los objetivos básicos de la información financiera, al reflejar los cobros y pagos de la empresa durante el ejercicio, con el objetivo de proporcionar información útil sobre sus actividades dirigidas a la obtención de efectivo o tesorería, para: la devolución de la deuda, las actividades de inversión y para pagar dividendos a los accionistas. (p. 68).

En este informe se analiza, cuenta a cuenta, de dónde obtuvo el efectivo la empresa y cómo fue utilizado. Permite también visualizar de forma ordenada las actividades en las que hubo movimientos de efectivo en lo operativo, lo que corresponde a financiamiento y en lo de inversión.

Por último, dentro de los informes contables y financieros las empresas deben adicionar una memoria. Para Juez Martel \& Martín Molina (2007), "la Memoria, completa, amplia, comenta y explica la información contenida en el Balance de Situación y en la cuenta de Pérdidas y Ganancias, además de cuántas informaciones tengan una sustantividad propia". (p. 58). El objetivo de la memoria es explicar con detalle cada una de las cuentas contenidas en el balance general y en las cuentas de pérdidas y ganancias. Además, la memoria debe contemplar la actividad de la empresa, su naturaleza y las políticas de registro contable aplicadas.

Los cinco informes mencionados permiten establecer parámetros de comparabilidad y lenguaje común para quienes generan y utilizan la información contable para divulgar información y 
tomar decisiones en lo interno y lo externo de la empresa. No obstante, cuando se habla de informes y de un lenguaje común en materia contable es necesario que los usuarios de la información contable, en este caso los directores y tomadores de decisiones de las pymes, tengan habilidades técnicas para la lectura de esos informes, y para interpretarlos y tomar decisiones con base en ellos.

Respecto a las habilidades técnicas, estas son todos los conocimientos que el recurso humano que labora en las pymes tenga de los conceptos y criterios contables, que permitan comprender los informes contables y financieros. Las habilidades técnicas son también los conocimientos y las competencias en un campo especializado, en este caso los conocimientos de contabilidad. (Robbins $\&$ Coulter, 2005, p. 12).

\section{Las habilidades técnicas exponen los} conocimientos adquiridos y la pericia necesaria para realizar acciones que incluyen: métodos, procesos y procedimientos. En lo contable, dichas habilidades requieren que se tenga conocimiento de la operatividad y funcionalidad de determinadas herramientas, sistemas y técnicas. Todo es un proceso integral que proporciona información cuya finalidad es servir de base para el control y la toma de decisiones.

El control es un paso del proceso administrativo que permite, con base en resultados, tomar decisiones en pro de mejorar los procesos. Para
Horngren, Sundem \& Stractton, Contabilidad Administrativa (2006), "la toma de decisiones es la elección con propósito definido hecha entre un conjunto de cursos de acción alternativos diseñados para alcanzar algún objetivo. Es el núcleo del proceso de administración”. (p. 12).

Sin embargo, es importante destacar que la toma de decisiones va de la mano con el proceso de control. Cuando se controla se pueden tomar decisiones porque se pueden comparar resultados propuestos con resultados dados u obtenidos. Por ende, el tomar decisiones es emprender acciones relacionadas con los resultados previstos en la fase de control; pero para ello se deben tener bases justificantes que permitan controlar. En este caso se estaría hablando de la información contable de primera mano y disponible en tiempo y espacio.

\section{MATERIALES Y MÉTOdoS}

El tipo de investigación utilizado es de carácter exploratorio-descriptivo. Con ella se pretende tener una visión general que permita aproximarse a una realidad específica de las pymes. Como metodología de investigación se seleccionaron aspectos vinculantes de cada una de las variables en estudio, y posteriormente se analizan y presentan resultados independientes. (Díaz Narváez , 2009, p. 180). Para recolectar la información se utilizó una encuesta que fue aplicada a 61 pymes. Dicha herramienta responde a lo que señala García Córdoba (2004), que se característica por contener "preguntas claras y 
concretas, presentadas en orden rígido $\mathrm{y}$ preestablecido que no puede alterarse; ello generará respuestas cortas y de contenido limitado". (p. 23). Este instrumento fue utilizado dadas las características estructurales de las pymes analizadas.

El muestreo seleccionado es de tipo intencional y responde a elementos muestrales de la población seleccionada a juicio del investigador. (Mohammad Naghi, 2005, p. 189). Con base en este razonamiento la escogencia de las pymes se hizo bajo dos criterios de selección: el primero, la facilidad de acceso y localización, y el segundo por ser empresas que contratan servicios contables independientes.
El trabajo de campo se realizó entre el 15 de enero y el 30 de octubre del 2015. A continuación, se exponen los resultados:

\section{RESULTADOS Y DISCUSIÓN}

\section{Tipos de actividad comercial y actividad económica}

En el cuadro $\mathrm{N}^{\circ} 1$ se establece un orden, una clasificación de las pymes estudiadas según el tipo de actividad que realizan y el tipo de actividad económica al que corresponde esa actividad. Nótese que todas realizan actividades económicas de servicios o de comercio y que según esas clasificaciones todas pertenecen al sector terciario, pues ninguna transforma materia prima.

\section{Cuadro $\mathbf{N}^{\circ} \mathbf{1}$}

Tipo de actividad económica que realizan las pymes

\begin{tabular}{lccc}
\hline \multicolumn{1}{c}{ Tipo de actividad } & Cantidad & Actividad económica & Sector \\
\hline Joyería & 1 & Comercial & Terciario \\
Venta de uniformes & 1 & Comercial & Terciario \\
Servicios de jardinería & 1 & Servicios & Terciario \\
Fotocopiadora & 1 & Servicios & Terciario \\
Cancha sintética & 1 & Servicios & Terciario \\
Bailes típicos & 2 & Servicios & Terciario \\
Desarrollo de Software & 2 & Servicios & Terciario \\
Alquiler de locales & 2 & Servicios & Terciario \\
Venta de artículos de computación & 2 & Comercial & Terciario \\
Panaderías & 3 & Comercial & Terciario \\
Heladerías & 3 & Comercial & Terciario \\
Peluquerías & 3 & Servicios & Terciario \\
Servicio de transporte & 3 & Servicios & Terciario
\end{tabular}




\begin{tabular}{lccc}
\hline \multicolumn{1}{c}{ Tipo de actividad } & Cantidad & Actividad económica & Sector \\
\hline Venta de alimentos & 3 & Comercial & Terciario \\
Servicios mecánicos & 3 & Servicios & Terciario \\
Pulpería & 4 & Comercial & Terciario \\
Veterinaria & 4 & Comercial & Terciario \\
Venta de lotería & 5 & Comercial & Terciario \\
Servicios de construcción & 5 & Servicios & Terciario \\
Servicios legales & 5 & Servicios & Terciario \\
Soda & 7 & Comercial & Terciario \\
\hline
\end{tabular}

Fuente: Consulta a pymes que contratan servicios contables.

Del total de 61 pymes encuestadas 54\% realizan tienen como actividad económica los servicios y $46 \%$ pertenecen a la actividad económica comercial.

\section{Cuadro $\mathrm{N}^{\circ} 2$}

Clasificación según tipo de actividad económica

\begin{tabular}{lcccc}
\hline \multicolumn{1}{c}{ Tipo de actividad } & Cantidad & $\begin{array}{c}\text { Actividad } \\
\text { económica }\end{array}$ & $\begin{array}{c}\text { Relativo por } \\
\text { actividad }\end{array}$ & $\begin{array}{c}\text { Relativo } \\
\text { acumulado }\end{array}$ \\
\hline Joyería & 1 & Comercial & $2 \%$ & $2 \%$ \\
Venta de uniformes & 1 & Comercial & $2 \%$ & $3 \%$ \\
Venta de artículos de & 2 & Comercial & $3 \%$ & $7 \%$ \\
computación & 3 & Comercial & $5 \%$ & $11 \%$ \\
Panaderías & 3 & Comercial & $5 \%$ & $16 \%$ \\
Heladerías & 3 & Comercial & $5 \%$ & $21 \%$ \\
Venta de alimentos & 4 & Comercial & $7 \%$ & $28 \%$ \\
Pulpería & 4 & Comercial & $7 \%$ & $34 \%$ \\
Veterinaria & 5 & Comercial & $8 \%$ & $43 \%$ \\
Venta de lotería & 7 & Comercial & $11 \%$ & $54 \%$ \\
Soda & 1 & Servicios & $2 \%$ & $56 \%$ \\
Servicios de jardinería & 1 & Servicios & $2 \%$ & $57 \%$ \\
Fotocopiadora & 1 & Servicios & $2 \%$ & $59 \%$ \\
Cancha sintética & 2 & Servicios & $3 \%$ & $62 \%$ \\
Bailes típicos & 2 & Servicios & $3 \%$ & $66 \%$ \\
Desarrollo de Software & 2 & Servicios & $3 \%$ & $69 \%$ \\
Alquiler de locales & & & &
\end{tabular}




\begin{tabular}{lcccc}
\hline \multicolumn{1}{c}{ Tipo de actividad } & Cantidad & $\begin{array}{c}\text { Actividad } \\
\text { económica }\end{array}$ & $\begin{array}{c}\text { Relativo por } \\
\text { actividad }\end{array}$ & $\begin{array}{c}\text { Relativo } \\
\text { acumulado }\end{array}$ \\
\hline peluquerías & 3 & Servicios & $5 \%$ & $74 \%$ \\
Servicio de transporte & 3 & Servicios & $5 \%$ & $79 \%$ \\
Servicios mecánicos & 3 & Servicios & $5 \%$ & $84 \%$ \\
Servicios de construcción & 5 & Servicios & $8 \%$ & $92 \%$ \\
Servicios legales & 5 & Servicios & $8 \%$ & $100 \%$ \\
\hline TOTALES & $\mathbf{6 1}$ & & $\mathbf{1 0 0 \%}$ & \\
\hline
\end{tabular}

Fuente: Consulta a pymes que contratan servicios contables.

\section{Servicio e información contable}

Como se indicó anteriormente, las pymes consultadas son empresas que contratan los servicios contables a oficinas de contabilidad. Consultadas sobre los motivos por los cuales contratan los servicios contables hubo respuestas variadas. El objetivo de esta pregunta era poder tener criterios que relacionen la información contable de las pymes con las decisiones que toman. No obstante, según las respuestas de los 61 consultados, 45 de ellos contratan los servicios de contabilidad para la presentación de los reportes tributarios. En el cuadro N. 3 se resumen los resultados de las consultas:

\section{Cuadro $\mathbf{N}^{\circ} 3$}

Motivos por los cuales las pymes contratan servicios contables

\begin{tabular}{lcc}
\hline Respuestas & Frecuencia de respuesta & Frecuencia relativa \\
\hline 1. Para presentar reportes tributarios & 45 & $74 \%$ \\
2. Para visualizar ganancias y pérdidas. & 22 & $36 \%$ \\
3. Por orden y control & 20 & $33 \%$ \\
4. Para toma de decisiones empresariales & 17 & $28 \%$ \\
5. Para presentación a instancias fuera de la empresa. & 16 & $26 \%$ \\
\hline
\end{tabular}

Fuente: Consulta a pymes que contratan servicios contables.

Los datos del cuadro $\mathrm{N}^{\circ} 3$ presentan las respuestas que podrían estar relacionadas con la contratación de servicios contables por parte de las pymes. En orden de importancia son: la presentación de reportes tributarios, visualización de ganancias y pérdidas, orden y control, toma de decisiones y presentación a instancias fuera de la empresa. Sin embargo, el motivo principal para la contratación 
de servicios según los resultados son los reportes y trámites tributarios, y $74 \%$ contratan los servicios contables para trámites relacionados con la presentación de reportes tributarios.

\section{Cuadro $\mathbf{N}^{\circ} 4$}

Tipo de contabilidad según el motivo de la contratación de los servicios

\begin{tabular}{lccc}
\hline \multicolumn{1}{c}{ Respuestas } & $\begin{array}{c}\text { Frecuencia de } \\
\text { respuesta }\end{array}$ & $\begin{array}{c}\text { Frecuencia } \\
\text { relativa }\end{array}$ & $\begin{array}{c}\text { Tipo de } \\
\text { contabilidad }\end{array}$ \\
\hline 1. Presentación de reportes tributarios & 45 & $74 \%$ & Fiscal \\
4. Para visualizar ganancias y pérdidas & 22 & $36 \%$ & Administrativa \\
2. Por orden y control. & 20 & $33 \%$ & Administrativa \\
3. Para toma de decisiones empresariales & 17 & $28 \%$ & Administrativa \\
5. Para presentación a instancias fuera de la & 16 & $26 \%$ & Financiera \\
empresa. & & &
\end{tabular}

Fuente: Dueños de pymes consultados en oficina de contabilidad Santa Cruz.

El cuadro $\mathrm{N}^{\circ} 4$ muestra la relación entre los tipos de contabilidad y los motivos por los cuales las pymes contratan los servicios contables. Según los datos obtenidos y las definiciones de los tipos de contabilidad, $74 \%$ de las pymes hacen uso de la contabilidad fiscal, pues lo que motiva la contratación es hacer gestiones tributarias. No obstante, en ningún resultado se nota un interés marcado o datos contundentes que indiquen que la información contable se utiliza para tomar decisiones dentro de la empresa. Por tanto, la contabilidad administrativa y la contabilidad financiera, en el caso de las pymes, son poco aplicables. Eso demuestra la poca relación que tiene la contabilidad que contratan las pymes con la toma de decisiones empresariales.
Respecto a la contabilidad financiera, por lo general cuando se deben hacer presentaciones fuera de la empresa, ya sea en bancos, a proveedores o en instituciones de gobierno, los reportes deben responder a las técnicas de la contabilidad financiera, pues esta tiene formas y bases establecidas según normas internacionales. En relación con este tipo de contabilidad, 26\% de las pymes encuestadas realizan alguna gestión fuera de la empresa; sin embargo, son situaciones esporádicas que no corresponden a las actividades cotidianas. Por lo tanto, no cumple con la condición de ser una herramienta para la toma de decisiones dentro de la empresa. 


\section{Capacidades técnicas}

Para el análisis de la variable "capacidades técnicas" se plantearon cinco preguntas, todas enfocadas en medir las capacidades que tienen las pymes para analizar y comprender los informes y aspectos contables dentro de la organización. Este vínculo sirve de parámetro para reforzar el argumento de por qué las pymes contratan la contabilidad en lugar de generarla internamente. A continuación, se presentan las respuestas a cada pregunta.

\section{Cuadro $\mathbf{N}^{\circ} 5$}

Formación académica de representantes de pymes en temas de contabilidad

\begin{tabular}{|l|l|c|c|}
\hline \multicolumn{1}{|c|}{ Ítems } & \multicolumn{1}{c|}{ Criterio } & Cantidad & Porcentaje \\
\hline $\begin{array}{l}\text { Formación académica relacionada con } \\
\text { la contabilidad }\end{array}$ & No tiene & 42 & $69 \%$ \\
\hline & Técnico & 5 & $8 \%$ \\
\hline & Bachiller en Contabilidad & 3 & $5 \%$ \\
\hline & Licenciatura en Contabilidad & 1 & $2 \%$ \\
\hline & Capacitaciones INA & 6 & $10 \%$ \\
\hline & Otros & 4 & $7 \%$ \\
\hline & Total & $\mathbf{6 1}$ & $\mathbf{1 0 0 \%}$ \\
\hline
\end{tabular}

Fuente: Pymes consultadas que contratan servicios contables.

Respecto de la pregunta sobre la formación académica relacionada con la contabilidad casi $70 \%$ de las pymes no tienen ninguna. Únicamente en cuatro de ellas dijeron tener estudios formales en contabilidad y en 15 tienen capacitación, aunque no suficientes como para establecer indicios del conocimiento base necesario.

Las respuestas a las otras cuatro preguntas se resumen en el cuadro $\mathrm{N}^{\circ} 6$. 


\section{Cuadro $\mathbf{N}^{\circ} 6$}

Conocimiento de paquetes contables, estados financieros básicos, interpretación de estados financieros y paquetes computacionales de las pymes

\begin{tabular}{|c|c|c|c|}
\hline Pregunta & Criterio & Cantidad & Porcentaje \\
\hline \multicolumn{4}{|l|}{ Conocimiento y manejo de } \\
\hline \multirow[t]{3}{*}{ paquetes contables } & No tiene & 54 & $89 \%$ \\
\hline & Sí tiene & 7 & $11 \%$ \\
\hline & Total & 61 & $100 \%$ \\
\hline \multicolumn{4}{|l|}{ Conocimiento de los estados } \\
\hline \multirow[t]{3}{*}{ financieros básicos } & No tiene & 37 & $61 \%$ \\
\hline & Sí los conoce & 24 & $39 \%$ \\
\hline & Total & 61 & $100 \%$ \\
\hline \multicolumn{4}{|c|}{ Conocimientos para interpretar } \\
\hline \multirow[t]{3}{*}{ estados financieros } & No puede interpretar & 43 & $70 \%$ \\
\hline & Sí los puede interpretar & 18 & $30 \%$ \\
\hline & Total & 61 & $100 \%$ \\
\hline \multicolumn{4}{|l|}{ Conocimiento y manejo de } \\
\hline \multirow[t]{3}{*}{ paquetes computacionales } & No tiene & 28 & $46 \%$ \\
\hline & Sí tiene & 33 & $54 \%$ \\
\hline & Total & 61 & $100 \%$ \\
\hline
\end{tabular}

Fuente: Pymes consultadas que contratan servicios contables.

A la pregunta sobre manejo y conocimiento de paquetes contables en $89 \%$ de las pymes respondieron negativamente. Únicamente en $7 \%$ de ellas dijeron tener conocimiento al respecto.

A la pregunta de si tienen conocimiento de lo que son estados financieros básicos 39\% respondieron afirmativamente; sin embargo, $61 \%$ dijeron no conocerlos. También se les consultó si sabían interpretar los estados financieros y $70 \%$ respondieron que no. Por último, se les preguntó si tienen conocimiento y saben utilizar paquetes computacionales y las respuestas estuvieron muy parejas: $46 \%$ indicaron no tener esos conocimientos y 54\% dijeron que sí.

En el análisis de esta variable se toman en consideración varios puntos. El primero de ellos 
es el de la capacitación, tema tratado en muchas investigaciones sobre pymes pero que sigue siendo preocupante porque los resultados reflejan una marcada serie de debilidades de tipo administrativo.

No obstante, aquí también queda claro que, en el aspecto de mercado, a pesar de que el Gobierno acepta abiertamente que las pymes son contribuyentes importantes en la producción interna del país, estas siguen tratando de sobrevivir con muy pocos recursos técnicos, sin apoyo, y llevándose muchos golpes que el mercado les da.

Otro de los aspectos por tener en cuenta es el relacionado con el uso que se le da a la información contable. En el nivel empresarial no se le saca el provecho necesario, y la razón principal es que las pymes no cuentan con la debida capacitación para hacerlo. Desde esta perspectiva interesa indicar que muchas de las pymes les dan principal importancia a las presiones ejercidas en cuanto a presentación de impuestos e informes tributarios. Por lo tanto, en lo contable las pymes ven su prioridad en la presentación de impuestos más que en la información disponible para tomar decisiones. Por la falta de capacitación y conocimiento de las bases contables las pymes deben recurrir a contratar los servicios externamente y, como consecuencia, ese apoyo externo altera uno de los objetivos principales de la contabilidad, el cual es servir de apoyo y control para que se tomen mejores decisiones empresariales.

\section{Criterios para contratar servicios contables}

Para el objetivo relacionado con los criterios que se utilizan en las pymes al contratar los servicios contables se plantearon cinco preguntas con respuestas abiertas. La finalidad era buscar respuestas relativas a por qué las pymes prefieren contratar servicios contables en lugar de llevar sus propias contabilidades. La primera de esas preguntas era sobre las características que deben tener las pymes para la contratación de sus servicios contables. Los resultados se encuentran referenciados en el cuadro $\mathrm{N}^{\circ} 7$. 


\section{Cuadro $\mathbf{N}^{\circ} 7$}

Características que debe tener un contador para ser contratado por las pymes

\begin{tabular}{|c|c|c|c|}
\hline Criterios & Ítems & Absoluto & Relativo \\
\hline Precio & & 52 & $85 \%$ \\
\hline Confianza & & 50 & $82 \%$ \\
\hline Puntualidad & & 39 & $64 \%$ \\
\hline Orden & & 30 & $49 \%$ \\
\hline Credibilidad & & 26 & $43 \%$ \\
\hline Honestidad & & 19 & $31 \%$ \\
\hline Ética profesional & & 17 & $28 \%$ \\
\hline Profesionalismo & & 15 & $25 \%$ \\
\hline Otros. & & 12 & $43 \%$ \\
\hline Total & & 61 & \\
\hline
\end{tabular}

Fuente: Pymes consultadas que contratan servicios contables.

Las características que favorecen la contratación de los contadores por las pymes son variadas. De ellas, el precio y la confianza son las de mayor representatividad; pero, con excepción del precio, todas las razones mencionadas como características para contratar a un contador tienen un componente de confianza. Por su naturaleza, las pymes necesariamente buscan a alguien en quien poder confiar y que las pueda apoyar en todas sus inquietudes, y también darles soporte en todos los trámites contables que deban realizar.

En cuanto a la pregunta sobre la importancia de llevar contabilidades las respuestas se resumen en el cuadro $\mathrm{N}^{\circ} 8$, enfocadas principalmente en dos razones fundamentales. La primera tiene que ver con presentar el reporte y pagar los impuestos; la segunda con evitar multas. El hecho de que los dueños de pymes piensen en la contabilidad como un proceso relacionado con los impuestos les limita la importancia que tiene llevarlas por orden, disciplina, y para toma de decisiones dentro de la empresa. Si se comparan estas respuestas con las del cuadro $\mathrm{N}^{\circ} 7$, en donde se visualiza que las características para contratar a un contador son el precio, la puntualidad y la confianza, estas razones están estrechamente ligadas a la necesidad de contar con alguien que les pueda ayudar en la presentación de los reportes tributarios, pero que les garantice hacerlo a tiempo y a un precio módico para la empresa. 


\section{Cuadro $\mathbf{N}^{\circ} 8$}

Importancia de llevar contabilidad para las

\begin{tabular}{|c|c|c|}
\hline \multicolumn{3}{|c|}{ pymes } \\
\hline Criterios & Absoluto & Relativo \\
\hline Presentar y pagar impuestos & 57 & $93 \%$ \\
\hline Evitar multas & 35 & $57 \%$ \\
\hline Orden y control & 28 & $46 \%$ \\
\hline Saber si hay pérdidas o & & \\
\hline ganancias & 20 & $32 \%$ \\
\hline Otros & 19 & $31 \%$ \\
\hline Total & 61 & \\
\hline
\end{tabular}

Fuente: Pymes consultadas que contratan servicios contables.

Como respuesta a la pregunta sobre el acceso a la información contable, en el cuadro $\mathrm{N} .^{\circ} 9$ se presentan los datos en los cuales la mayoría de las pymes dijeron que el director y el contador; sin embargo, esto no es suficiente para saber si ese acceso favorece el análisis y la toma de decisiones, o si más bien es un asunto de secreto profesional o de discreción.

\section{Cuadro $\mathbf{N}^{\circ} 9$}

\begin{tabular}{lcc}
\multicolumn{3}{c}{$\begin{array}{c}\text { ¿Quiénes tienen acceso a la información contable de } \\
\text { las pymes? }\end{array}$} \\
\hline Criterios & Ítems & Relativo \\
\hline Contador y Dirección & 24 & $39 \%$ \\
Dirección & 19 & $31 \%$ \\
Colaboradores & 13 & $21 \%$ \\
Todos & 5 & $8 \%$ \\
\hline Total & $\mathbf{6 1}$ & $\mathbf{1 0 0 \%}$ \\
\hline
\end{tabular}

Fuente: Pymes consultadas que contratan servicios contables.

Según los datos del cuadro N. ${ }^{\circ} 10$, los motivos para que la empresa no haga sus propias contabilidades son: falta de capacitación del personal que labora en ellas y de presupuesto para poder contratar a un contador de planta. Otros motivos, como el desconocimiento, también se podrían clasificar como falta de capacitación, lo que aumenta el número de opiniones que son indicios de las necesidades de capacitación que aún presentan las pymes. En lo que respecta al presupuesto, la respuesta estuvo muy relacionada con la que dieron cuando se les preguntó sobre las características que debe tener un contador para ser contratado por las pymes, y a lo cual respondieron que el precio. Esto último confirma la intención de las pymes de pagar precios bajos por los servicios contables. 


\section{Cuadro $\mathbf{N}^{\circ} \mathbf{1 0}$}

Motivos por los que las pymes no llevan sus propias contabilidades

\begin{tabular}{|c|c|c|}
\hline Criterios & Absoluto & Relativo \\
\hline Falta de personal capacitado & 37 & $61 \%$ \\
\hline Falta de presupuesto para & & \\
\hline contratar a un contador & 17 & $28 \%$ \\
\hline Desconocimiento & 7 & $11 \%$ \\
\hline Total & 61 & \\
\hline
\end{tabular}

Fuente: Pymes consultadas que contratan servicios contables.

\section{CONCLUSIONES}

\section{Tipos de actividad comercial y actividad económica}

Los tipos de actividad comercial que realizan las pymes consultadas son la comercial y la de servicios; ninguna de ellas la industrial. El hecho de que las empresas industriales deban llevar controles minuciosos en sus procesos podría ser la razón por la cual este tipo de empresas no contratan los servicios contables de un despacho o contador.

Las actividades económicas de todas las pymes estudiadas están clasificadas como del sector terciario pues todas realizan actividad comercial o de servicios.

\section{Servicio e información contable}

Las pymes contratan los servicios contables principalmente para la elaboración de los informes tributarios que deben presentar.

Otra de las razones, pero menos importante, para que las pymes contraten servicios contables es la necesidad de visualizar los resultados operativos de la empresa; no obstante, la importancia que se le da a esto no es suficiente para creer que es para la toma de decisiones empresariales.

Las principales razones por las cuales las pymes demandan servicios contables de terceros tienen que ver con la presentación de informes y con reportes tributarios, y para visualizar pérdidas y ganancias que genera la empresa. Las razones vinculadas con la toma de decisiones son el orden y el aseo, y la toma de decisiones propiamente; pero ambas razones están por debajo de $50 \%$ de interés.

\section{Capacidades técnicas}

Las pymes no tienen la formación académica necesaria para hacerles frente a los procesos contables que las empresas ameritan, por lo tanto, no hay capacidad técnica para utilizar la información contable en la toma de decisiones empresariales. 
El nivel de capacitación de las pymes en temas relacionados con la contabilidad es muy bajo pues casi $70 \%$ de ellas no lo tienen.

En materia de interpretación de estados financieros más de la mitad de las pymes consultadas no saben interpretar los datos contenidos en los informes financieros.

En las pymes hay un conocimiento generalizado de lo que son los estados básicos de la contabilidad, pero no saben interpretar lo que en realidad dicen; por ende, el análisis de información contable no forma parte de la toma de decisiones dentro de la empresa.

Las pymes que contratan servicios contables no tienen en sus empresas sistemas contables que ayuden en los procesos, por lo cual el conocimiento queda en manos del contador o de la oficina de contabilidad.

\section{Criterios para contratar servicios contables}

Para contratar servicios contables los propietarios de pymes toman en cuenta los precios, la confianza y la puntualidad.

Los servicios contables contratados son para la presentación oportuna de los reportes de impuestos para evitar así multas. En ambos casos la razón principal es la presentación de los reportes tributarios.
Los propietarios de pymes tienen acceso a la información contable pero ese acceso no tiene relación con la utilidad que se le da para tomar decisiones en el ámbito empresarial.

Las pymes no llevan sus propias contabilidades debido a la falta de capacitación y tampoco contratan a un contador de planta por problemas de presupuesto.

Finalmente, se evidencia que la mayoría de las pymes que contratan servicios de oficinas o despachos para que les lleven la contabilidad no aprovechan la información contable ampliamente, pero sí lo hacen básicamente para la presentación de reportes de impuestos. La información no está disponible en el momento indicado y no hay evidencia de que las decisiones gerenciales y administrativas se tomen con base en los reportes.

Como conclusión general habría que hacer un análisis o estudio detallado de los costos y beneficios que significaría culturizar a las pymes para que creen sus propias contabilidades y que sean quienes las administran y coordinen. Sin embargo, sin entrar en detalles de los pros y los contras, se sabe que el llevar contabilidades generadas en lo interno de la empresa permite tener información inmediata, hacer cálculos y luego compararlos con datos obtenidos por otras empresas de la industria. Además, habría un beneficio para las instituciones y entes reguladores porque ellos tendrían información de primera 
mano de las pymes y así podrían tomar decisiones acertadas con agilidad y precisión.

\section{REFERENCIAS}

Alcarria Jaime, J. J. (2009). Contabilidad Financiera I. España: Publicaciones de la Universitat Jaume.

Alvira Martín, F. (2011). La encuesta: una perspectiva general metodológica. Madrid, España: Centro de Investigaciones Sociológicas.

Ayllón Torres, M. T. (2004). Geografía Económica. México: Limusa.

Colegio de Contadores Privados de Costa Rica. (28 de enero de 2015). Incorporaciones. Obtenido de Incorporaciones: http://www.contador.co.cr

Colegio de Contadores Públicos de Costa Rica. (22 de agosto de 1947). Ley del Colegio de Contadores Públicos de Costa Rica. San José, San José, Costa Rica.

Díaz Narváez, V. P. (2009). Metodología de la investigación científica y bioestadística. Santiago, Chile: Ril Editores.

Eslava, J. d., \& Marín, I. d. (2009). Las claves de la nueva contabilidad para las Pymes. Madrid, España: Esic Editorial.
García Córdoba, F. (2004). El cuestionario. México: Limusa.

Horngren, C. T. (2003). Contabilidad. México: Pearson Educación.

Horngren, C. T., Datar, S. M. \& Foster, G. (2007). Contabilidad de Costos. Un enfoque gerencial. México: Pearson Educación.

Horngren, C. T., Sundem, G. L., \& Elliot, J. A. (2000). Introducción a la Contabilidad Financiera. México: Prentice Hall.

Horngren, C. T.; Sundem, G. L. \& Stractton, W. O. (2006). Contabilidad Administrativa. México: Pearson Educación.

Juez Martel, P., \& Martín Molina, P. B. (2007). Manual de Contabilidad para Juristas: Análisis y valoración de empresas. Madrid, España: La Ley.

Martínez, R. (2009). Manual de Contabilidad para Pymes. Alicante: Editorial Club Universitario.

Mohammad Naghi, N. (2005). Metodología de la Investigación. México: Limusa.

Naciones Unidas. (2002). Clasificación Industrial Internacional Uniforme. Nueva York, Estados Unidos de América: Naciones Unidas. 
Namakforoosh, M. (2005). Metodología de la Investigación. México: Limusa.

Robbins, S. P. \& Coulter, M. (2005). Administración. México: Pearson Educación.

Robles Valdés, G. \& Alcérreca Joaquín, C. (2000).

$\begin{array}{lcc}\text { Administración, } & \text { un } & \text { enfoque } \\ \text { interdisciplinario. } & \text { México: } & \text { Pearson } \\ \text { Prentice Hall. } & & \end{array}$

